\title{
Lesions in Nucleus Basalis Magnocellularis and Medial Septal Area of Rats Produce Qualitatively Similar Memory Impairments ${ }^{1}$
}

\author{
DAVID J. HEPLER, ${ }^{2}$ DAVID S. OLTON, GARY L. WENK, ${ }^{3}$ AND JOSEPH T. COYLE* \\ Department of Psychology, The Johns Hopkins University, Baltimore, Maryland 21218 and *Departments of Psychiatry, Neuroscience, \\ Pharmacology and Experimental Therapeutics, and Pediatrics, The Johns Hopkins University School of Medicine, \\ Baltimore, Maryland 21205
}

\begin{abstract}
The functional contribution of nucleus basalis magnocellularis (NBM) and the medial septal area (MSA) to memory was evaluated in two different spatial discriminations. Preoperatively, rats were trained to a criterion level of performance in a simultaneous left/right discrimination on the stem of a T-maze (a trial-independent memory) and a discretetrial, rewarded alternation discrimination on the arms of the T-maze (a trial-dependent memory). Bilateral lesions were made by injecting ibotenic acid (IBO) into the NBM, MSA, both NBM and MSA, or dorsal globus pallidus (DGP), and by radiofrequency current (RF) in the NBM and MSA. Control rats received operations in which either no current was passed or no neurotoxin was injected. Lesions in the NBM, MSA, or both the NBM and MSA produced a similar pattern of behavioral changes relative to the performance of controls; postoperative reacquisition of the arm discrimination was initially impaired but showed recovery to normal levels, whereas postoperative reacquisition and reversal of the stem discrimination was not impaired (except following the combined NBM and MSA lesion). Lesions of the DGP had no effect on choice accuracy in any discrimination. When the discrimination on the arms was made more difficult by increasing the delay interval during which the information had to be remembered, rats with combined NBM and MSA lesions were again impaired relative to controls and showed no signs of recovery of function. These results provide information about the behavioral functions of the basal forebrain cholinergic system and suggest that pathological changes in certain components of this system can cause disorders of memory.
\end{abstract}

Within the basal forebrain of the rat is a core of acetylcholinergic (ACh) neurons (Sofroniew et al., 1982) that has been divided into several regions which include the nucleus basalis magnocellularis

Received February 28, 1984; Revised September 17, 1984:

Accepted September 28, 1984

\footnotetext{
${ }^{1}$ This research was supported by National Institute of Neurological and Communicative Disorders and Stroke Grant NS18414 and by the Department of Defense Grant DAMD 17-C-2225. We thank M. Shapiro, C. Wible, and B. Knowlton for critically reading the manuscript, $C$. Redding for histology, $L$. McCall, L. Baker, and B. Cribbs for technical assistance, and M. McKinney and J. Lehmann for preliminary data.

${ }^{2}$ Present address: E. I. DuPont de Nemours \& Co., Biomedical Products, Experimental Station, Building 400, Room 4420, Wilmington, DE 19898.

${ }^{3}$ To whom correspondence should be addressed.
}

(NBM), diagonal band of Broca, and the medial septal area (MSA) Lehmann et al., 1980; Johnston et al., 1981; Bigl et al., 1982; Hartgraves et al., 1982; Pearson et al., 1983; Saper, 1984). The NBM projects ACh afferents primarily to the neocortex, and the MSA and vertical limb of the diagonal band project ACh afferents primarily to the hippocampus.

The frontal neocortex and hippocampus, and the cholinergic systems afferent to these areas, have an important role in memory (Bartus, 1980; Bartus et al., 1982; Mishkin, 1982). Recently, the behavioral effects of lesions produced in either the NBM (LoConte et al., 1982; Flicker et al., 1983) or MSA (Mitchell et al., 1982) have been evaluated separately in individual tasks, but the behavioral effects of these two lesions have not been compared on the same tasks.

In the present study, both ibotenic acid (IBO) and radiofrequency (RF) current were used to produce lesions. IBO causes degeneration of neuronal perikarya at the site of injection, without damaging axons of passage or neurons distant from the injection site (Coyle and Schwarcz, 1983). RF lesions destroy both neuronal perikarya and fibers of passage. The comparison of RF and IBO lesions allows for the assessment of the relative importance of damage to the vell bodies, as compared to the axons passing through the lesioned area, for these behaviors.

IBO lesions were also made in the dorsal globus pallidus (DGP) to control for the possibility that the behavioral changes produced by IBO in the NBM and MSA were due to generalized effects of IBO rather than specific damage in the NBM and MSA. The DGP is anatomically close to the NBM and MSA and contains cholinergic neurons (Saper, 1984). These neurons do not project to the frontal cortex or hippocampus, and the DGP has not been linked to memory functions. If the behavioral changes following IBO lesions in the NBM and MSA are due to diffuse effects of IBO, then IBO lesions of the DGP should produce behavioral effects similar to those following IBO lesions in the NBM and MSA. If these behavioral changes following IBO injections in the NBM and MSA are due to specific damage to these particular structures, then IBO injections into the DGP should have no effect on performance in these tasks.

\section{Materials and Methods}

\section{Subjects}

The subjects were 94 male albino rats ( 350 to $400 \mathrm{gm}$ ) obtained from Charles River Breeding Laboratories. Each rat was housed individually throughout testing, with free access to water, and was maintained on a 16 $\mathrm{hr} / 8$-hr light/dark cycle with lights on at 7:00 A.M. Each rat was deprived to $85 \%$ of its ad libitum weight prior to shaping and was maintained at this weight, plus $5 \mathrm{gm}$ per week for growth, throughout the experiment. At the completion of the day's testing, each rat was fed the appropriate amount of Charles River Rat Formula 


\section{Apparatus}

Two identical wooden T-mazes (Fig. 1) were used. On either side of the starting platform, stem, and arms were wooden edges, $2.0 \mathrm{~cm}$ high. The starting platform was separated from the stem by a guillotine door, $11.5 \mathrm{~cm}$ high and $6.2 \mathrm{~cm}$ wide, mounted in a frame, $22.5 \mathrm{~cm}$ high and $15.0 \mathrm{~cm}$ wide. The stem was divided into two halves by a hardware cloth partition, $12.0 \mathrm{~cm}$ high and $31.5 \mathrm{~cm}$ long, which started $24.0 \mathrm{~cm}$ from the guillotine door and was $24 \mathrm{~cm}$ long. At the end of the partition proximal to the arms, on both sides of the stem, was a white opaque curtain, $14.0 \mathrm{~cm}$ high and $7.0 \mathrm{~cm}$ wide, suspended from a frame, $14.0 \mathrm{~cm}$ high and $15.5 \mathrm{~cm}$ wide, which was perpendicular to the stem. A clear Plexiglas barrier, $14.0 \mathrm{~cm}$ high and 7.8 $\mathrm{cm}$ wide, was placed behind one curtain to block access to the arms on that side of the stem. A food cup, $1.0 \mathrm{~cm}$ wide and $1.5 \mathrm{~cm}$ deep, was located $1.0 \mathrm{~cm}$ from the distal end of each arm. The two mazes were located in different locations to provide unique extra-maze cues for each maze.

\section{Procedure}

\section{Shaping}

Each rat was trained for 5 days to run to the ends of the arms for a food reward (Thrive Cat Food, Purina). During the first day, food was liberally spread throughout the maze; each rat was allowed $20 \mathrm{~min}$ to explore. During each succesive day, the amount of food was progressively restricted so that, by the fifth day, food was present only in the food cups. Each rat received an equal amount of shaping at each of the two maze locations. The rats were given no food in their home cages during the shaping procedure and received only what food they ate during the daily shaping sessions.

\section{Preoperative testing}

The trial-independent memory discrimination (reference memory) consisted of a simultaneous left/right discrimination on the stem of each T-maze. One side of the hardware cloth partition was blocked by the Plexiglas barrier in one maze location, whereas the opposite side was blocked in the other maze location. In order to choose the unobstructed side of the stem, the rat had to determine the maze location in which it was being tested. A response was recorded for the stem discrimination when the rat placed its head more than $10 \mathrm{~cm}$ beyond the start of the hardware cloth barrier. If the rat chose the open side of the stem, a correct stem response was recorded and the rat continued to the arms. If the rat chose the blocked side of the stem, an incorrect stem response was recorded and the rat was allowed to turn around and choose the open side. The side that was blocked remained constant for any particular rat in all trials, but varied annong rats.

The trial-dependent memory discrimination (working memory) was a discrete-trial, rewarded alternation in the arms of the T-maze. Each trial consisted of a forced run, in which the rat was directed to one arm, and a choice run, in which the rat was able to choose either arm. At the start of

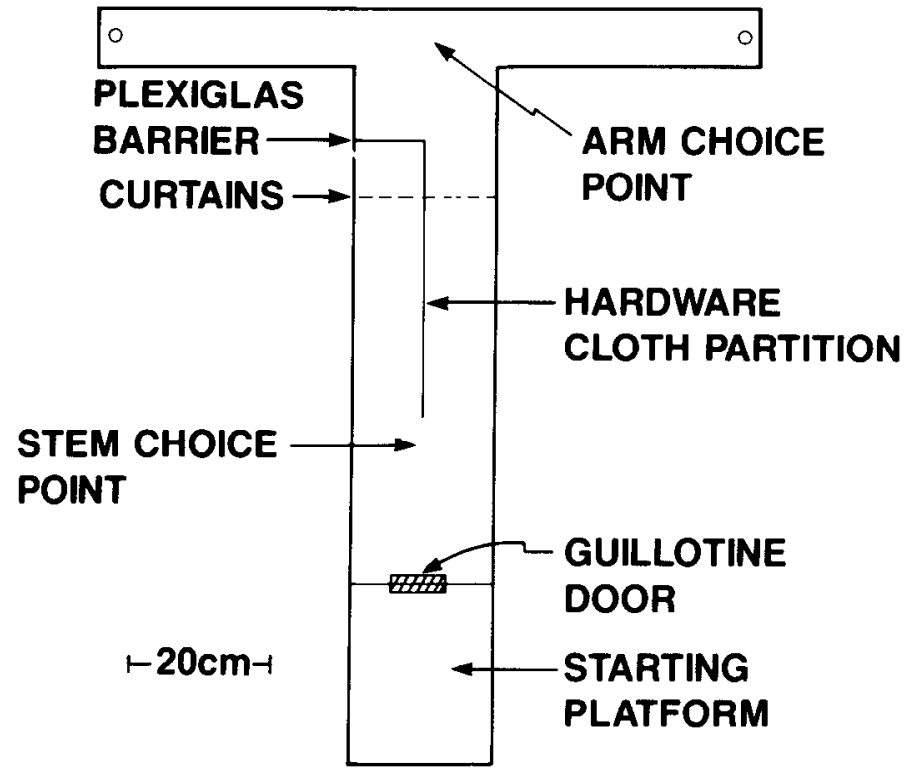

Figure 1. Scale drawing of the apparatus. See the text for detailed description. each trial, one piece of food was placed in the cup on the end of each of the two arms. During the forced run, a large piece of wood was used to block the entrance to one of the arms. The rat was placed on the starting platform and the guillotine door was raised, allowing the rat to run down the stem and enter the unblocked arm to get the reward. For the choice run, the block was removed and the rat was again placed on the starting platform. After a 5-sec delay, the guillotine door was raised and the rat was allowed to run down the stem and choose one of the arms. A response was recorded when the rat placed its head more than $10 \mathrm{~cm}$ down an arm. If the rat chose the arm which had been blocked during the forced run, a correct arm response was recorded. If the rat chose the arm to which it had been previously forced, an incorrect arm response was recorded. The rat was then returned to its home cage.

Once per day, 5 days each week, the rat was given a test session consisting of eight trials separated by an inter-trial interval of approximately $2 \mathrm{~min}$. The particular maze on which the rat was tested and the arm to which the rat was forced was varied pseudorandomly between trials so that, in each test session, four trials were given on each maze and each arm on each maze was blocked for the forced run of two trials. Also, the two $T$. mazes were interchanged approximately once each week to be certain that the rats were not using particular cues on the mazes to solve the task.

Preoperatively, each rat received at least 10 days of testing. Testing was continued until the rat reached a criterion of at least 7 correct responses in the 8 trials of the arm discrimination and at least 15 correct responses in the 16 trials of the stem discrimination each day for five consecutive days. Each rat was then assigned to one of three experimental groups for surgery.

\section{Experimental design}

Lesions were placed in the NBM and in the MSA by IBO and RF. These four groups are identified by a compound abbreviation indicating the lesion site and the type of lesion: NBM-IBO, NBM-RF, MSA-IBO, and MSA-RF. Lesions produced by IBO were also placed in both the NBM and MSA (NBM $+\mathrm{MSA}$ ) and in the DGP. One group of rats received operations but no lesion, and is identified by the abbreviation CON (control).

The experiment proceeded in four sections, each of which included a group of CON rats. The lesion groups in each section were: (1) NBM-RF, MSA-RF; (2) NBM-IBO. MSA-IBO; (3) NBM + MSA; and (4) DGP. An additional group of rats with $\mathrm{BBO}$ lesions in the NBM and their controls received no behavioral testing and were sacrificed 10 days after surgery for biochemical analysis.

\section{Surgery}

Prior to surgery, each rat received $0.3 \mathrm{ml}$ of $0.5 \mathrm{mg} / \mathrm{ml}$ of atropine methylbromide (Sigma Chemical Co., St. Louis, MO) intraperitoneally. Thirty minutes later, each rat was anesthelized with $0.3 \mathrm{ml} / \mathrm{kg}$ of Chloropent (Fort Dodge Laboratories, Fort Dodge, IA). The rat was placed in a David Kopf stereotaxic instrument with the incisor bar set so that bregma and lambda were in the same horizontal plane. The scalp was incised and retracted. Holes were drilled through the skull in the appropriate locations, and the lesion was made. The scalp was sutured, and the rat received $0.1 \mathrm{ml}$ of Bicillin (Wyeth Laboratories, Philadelphia, PA) intramuscularly in both hindlegs. The rat was then removed from the stereotaxic instrument and placed under a heat lamp until it awakened.

CON rats $(n=39)$ received the same surgical treatment as the lesioned rats except that no electrode or syringe needle was lowered into the brain.

NBM lesions $(n=28)$ were made at the following coordinates for both RF and IBO lesions: $0.8 \mathrm{~mm}$ posterior to bregma, $2.8 \mathrm{~mm}$ lateral to the central sinus, and $6.9 \mathrm{~mm}$ ventral from the dura. The rats received RF lesions in two stages: a unilateral lesion was produced, the rats were allowed 7 days to recover, and then the contralateral lesion was produced. RF lesions $(n=17)$ were produced by passing $16 \mathrm{~mA}$ of current (Grass Lesion Maker model LM 4, Grass Instruments, Quincy, MA) for $18 \mathrm{sec}$ through an electrode $0.2 \mathrm{~mm}$ in diameter, insulated except for $0.6 \mathrm{~mm}$ at the tip. Bilateral IBO lesions $(n=$ 11) were placed by injecting $25 \mathrm{nmol}$ of IBO (Sigma) in $1.0 \mu$ l of phosphatebuffered saline with a $1.0-\mu \mathrm{l}$ Hamilton syringe during $10 \mathrm{~min}$. The syringe was left in place for $5 \mathrm{~min}$ after the completion fo the infusion. The contralateral NBM then received a similar injection of IBO.

MSA lesions $(n=20)$ were made at the following coordinates for both the RF and IBO groups: $0.8 \mathrm{~mm}$ anterior to bregma, at the midline, and $5.5 \mathrm{~mm}$ ventral from the dura. RF lesions ( $n=10$ ) were produced by passing $12 \mathrm{~mA}$ of current for $14 \mathrm{sec}$ through an electrode $0.2 \mathrm{~mm}$ in diameter, insulated except for $0.6 \mathrm{~mm}$ at the tip. IBO lesions $(n=9)$ were produced by injecting $18 \mathrm{nmol}$ of IBO in $0.7 \mu \mathrm{l}$ of phosphate-buffered saline during a period of 5 $\min$. 
$\mathrm{NBM}+\mathrm{MSA}$ lesions $(n=9)$ were made at the same coordinates with $\mathrm{IBO}$ as described above. All injections were made in one surgical procedure. DGP lesions $(n=8$ ) were placed $0.4 \mathrm{~mm}$ posterior to bregma, $2.6 \mathrm{~mm}$ lateral to the central sinus, and $3.0 \mathrm{~mm}$ ventral from the dura by injecting 25 $\mathrm{nmol}$ of IBO in $1.0 \mu$ l of phosphate-buffered saline during a period of $5 \mathrm{~min}$.

\section{Postoperative testing}

Retention. Postoperatively, the test procedure was the same as that at the end of preoperative testing. Each rat was tested until it reached criterion.

Stem reversal. The stem discrimination was reversed. The Plexiglas barrier was moved to the opposite side of the stem of each maze. Thus, to gain access to the arms, the rat had to go down the side of each stem opposite that to which it had been trained preoperatively. With the exception of the reversal on the stem, the task was unchanged. Each rat was tested until it reached criterion.

Extended delay. Nine rats with NBM + MSA lesions and 10 CON rats continued testing after reaching criterion performance. For 6 test sessions, the delay between the forced and choice runs was $5 \mathrm{sec}$. For the next 18 test sessions, the delay was 10 min. For the next 6 test sessions, the delay was $5 \mathrm{sec}$. The rats were then sacrificed.

\section{Biochemical analyses}

Four untrained rats with NBM-IBO lesions and four rats without lesions were sacrificed 10 days after surgery to determine the effectiveness and specificity of the IBO lesions (Wenk et al., 1984). Each rat was decapitated and the brain was rapidly removed. Tissue samples (50 to $75 \mathrm{mg}$ ) were taken from areas 2 and 10 (according to the atlas of Krieg, 1946) of the frontolateral cortex and combined, and were taken separately from the dorsal and ventral hippocampus.

All other rats were sacrificed at the completion of behavioral testing, approximately 8 weeks after surgery. Tissue samples (50 to $75 \mathrm{mg}$ ) were taken from frontolateral cortex and the dorsal hippocampus. Choline acetyltransferase (ChAT) levels were measured by the method of Fonnum (1975). Protein was measured according to the method of Lowry et al. (1951). For both the IBO and RF sections of the experiment, all rats within each group were sacrificed within 3 days of each other.

\section{Histology}

After the removal of samples for biochemical analysis, the remaining brain tissue was fixed in a $10 \%$ formalin: $30 \%$ sucrose solution. The brain was frozen and sectioned coronally at $30 \mu \mathrm{m}$ with a frozen stage microtome. Every fifth section throughout the lesion site was mounted on a glass slide and stained with cresyl violet. The size and location of the lesions were determined by microscopic examination for loss of magnocellular neurons and the prosence of gliosis.

\section{Statistics}

The data were analyzed using an analysis of variance with post hoc Sheffe contrasts.

\section{Results}

\section{ChAT levels}

The levels of ChAT activity in the rats with lesions were compared to the values in rats with CON operations to determine the percentage decrease in activity caused by the lesion. The levels of ChAT activity for the rats with NBM-IBO lesions sacrificed 10 days after surgery are shown in Table I. IBO lesions decreased the levels of ChAT activity in the frontal cortex $(p<0.01)$ but did not affect levels of ChAT activity in either the dorsal or ventral hippocampus ( $p<$ $0.05)$.

The levels of ChAT activity in the frontal cortex and hippocampus of rats that received behavioral testing are summarized in Table II. For CON rats, the mean levels of ChAT activity of both the frontal cortex and hippocampus were significantly lower for the IBO section of the experiment than for the RF section $(p<0.01)$. Therefore, the data for the rats with lesions were compared to those of the CON rats in their own section. NBM lesions, produced by both IBO and RF, significantly decreased levels of ChAT activity in the frontal cortex but not in the dorsal hippocampus $(p<0.01)$. MSA lesions, produced by both $\mathrm{IBO}$ and RF, significantly decreased levels of
TABLE I

ChAT levels in frontal cortex, dorsal hippocampus, and ventral hippocampus for rats with lesions produced by $I B O$

\begin{tabular}{|c|c|c|c|c|}
\hline \multirow{3}{*}{ Group } & \multirow{3}{*}{$N$} & \multicolumn{3}{|c|}{ ChAT (nmol/mg of protein/hr) } \\
\hline & & \multirow{2}{*}{ Frontal Cortex } & \multicolumn{2}{|c|}{ Hippocampus } \\
\hline & & & Dorsal & Ventral \\
\hline Control & 4 & $22.70 \pm 0.61^{a}$ & $27.00 \pm 1.49$ & $30.85 \pm 1.04$ \\
\hline NBM & 4 & $11.78 \pm 2.06^{b}$ & $26.27 \pm 1.31$ & $30.20 \pm 1.47$ \\
\hline
\end{tabular}

${ }^{a}$ Values are mean \pm SEM.

${ }^{b} p<0.01$ compared to corresponding region of control rats using the Student's two-tailed $t$ test. These rats received no behavioral testing and were sacrificed 10 days after the lesions were placed.

\section{TABLE ॥}

ChAT levels in frontal cortex and dorsal hippocampus for rats with lesions produced by ibotenic acid (top) and radiofrequency current (bottom)

\begin{tabular}{llcc}
\multirow{2}{*}{ Group } & $N$ & \multicolumn{2}{c}{ ChAT (nmol/mg of protein/hr) } \\
\cline { 3 - 4 } & & Frontal cortex & Hippocampus \\
\hline Control & 26 & $22.85 \pm 1.49^{a}$ & $29.53 \pm 1.99$ \\
NBM & 7 & $9.91 \pm 2.36^{b}$ & $27.72 \pm 2.36$ \\
MSA & 7 & $24.47 \pm 2.10$ & $14.86 \pm 3.03^{b}$ \\
NBM + MSA & 9 & $14.10 \pm 1.16^{b}$ & $19.32 \pm 0.96^{b}$ \\
Control & \multicolumn{2}{c}{ Radiofrequency } \\
NBM & 13 & $31.94 \pm 1.07$ & $36.20 \pm 1.36$ \\
MSA & 12 & $24.03 \pm 2.10^{b}$ & $37.41 \pm 3.12$ \\
M & 10 & $28.91 \pm 2.01$ & $22.90 \pm 2.97^{b}$ \\
\hline
\end{tabular}

${ }^{a}$ Values are mean \pm SEM.

${ }^{b} p<0.01$ compared to corresponding region of control rats using the Student's two-tailed $t$ test.

ChAT activity in the hippocampus but not in the frontal cortex $(\rho<$ 0.01). NBM + MSA lesions significantly decreased levels of ChAT activity in both the frontal cortex and hippocampus $(p<0.01)$. DGP lesions did not significantly alter levels of ChAT activity in either the frontal cortex or the hippocampus (99 and 100\% of control regions, respectively).

\section{Histology}

Lesions in the NBM were centered in the substantia innominata for both the NBM-IBO and NBM-RF groups and extended $2.0 \mathrm{~mm}$ caudally from the anterior commissure. A typical set of lesions is shown schematically in Figure 2. IBO lesions in the MSA destroyed most of the cells of the medial septum and the dorsal part of the vertical limb of the diagonal band. RF lesions in the MSA destroyed only the MSA. Lesions in the DGP involved the most dorsal extent of the globus pallidus and striatum just below the corpus callosum (Fig. 3).

\section{Behavior}

\section{Preoperative}

Choice accuracy in both discriminations was near the level expected by chance for all rats at the start of preoperative testing. Within 10 preoperative test sessions, however, every rat performed at or above criterion levels in both the stem and arm discriminations.

\section{Postoperative}

After the second lesion, many of the rats in the NBM-IBO and NBM-RF groups showed normal posture but did not eat, drink, or groom. These rats were intubated and fed intragastrically (SMA, Wyeth; $10 \mathrm{ml}$ every $6 \mathrm{hr}$ ). Four rats with IBO lesions and five rats with $\mathrm{RF}$ lesions never recovered and subsequently died. Rats who did recover usually began eating about 1 week after the contralateral 
IBOTENIC ACID

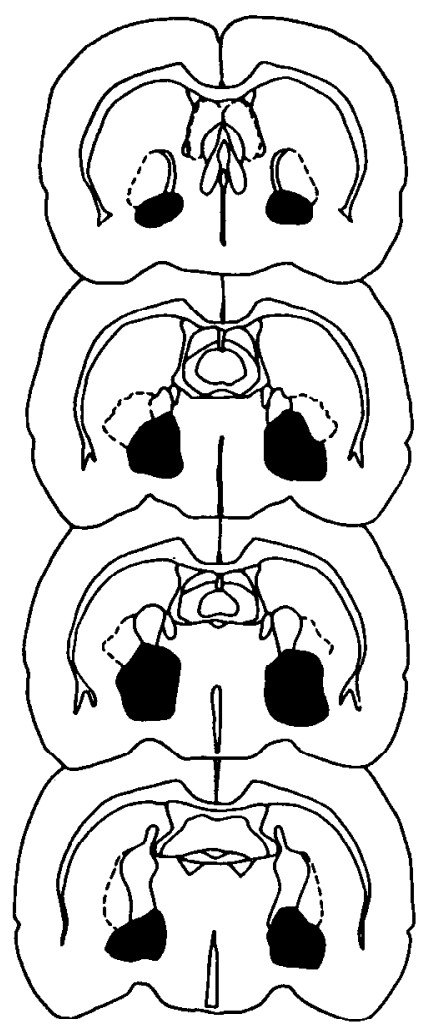

\section{RADIOFREQUENCY CURRENT}

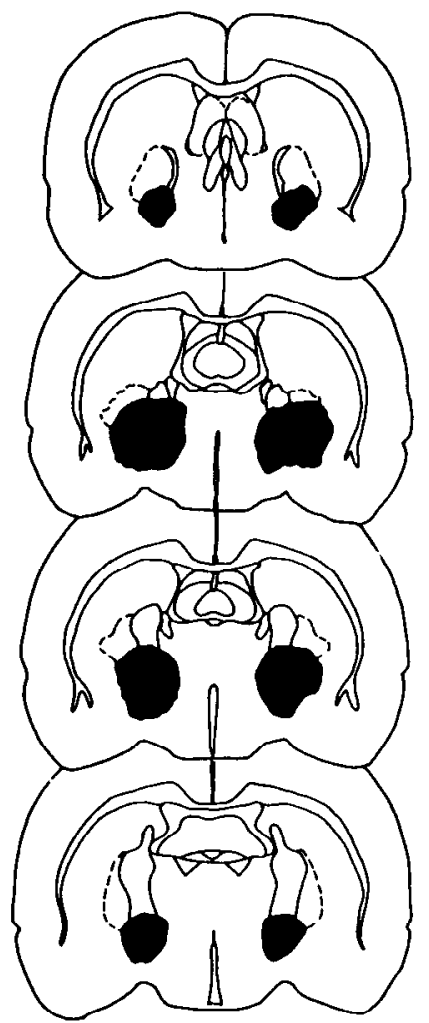

IBOTENIC ACID

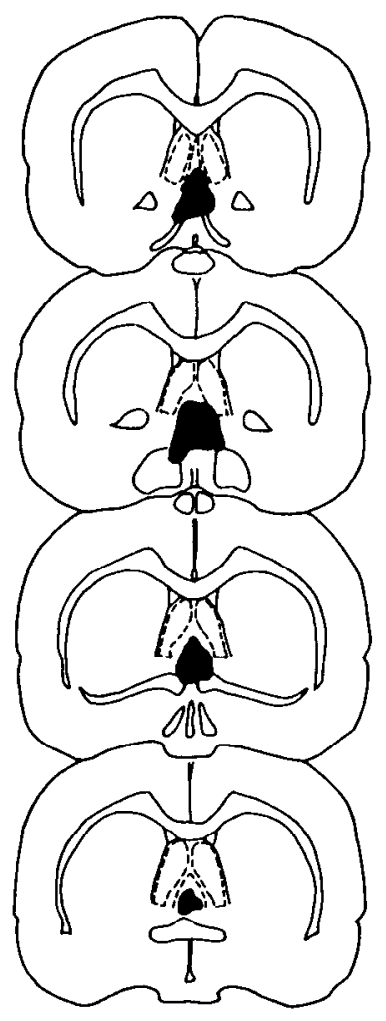

RADIOFREQUENCY CURRENT

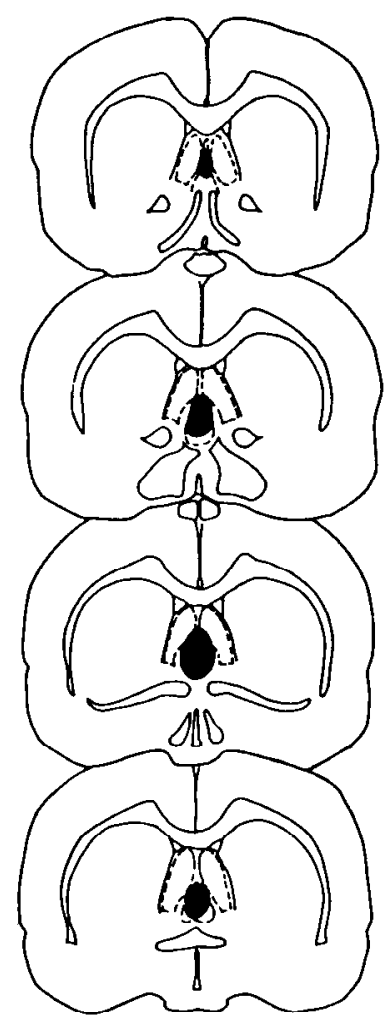

Figure 2. Drawings of coronal sections showing typical IBO and RF lesions in the NBM (left) and MSA (right). Shaded areas indicate areas of cell loss.

lesion. Rats in the MSA-IBO and MSA-RF groups were generally as healthy as the CON rats immediately after surgery. Two rats in the MSA-IBO group died several days later. All rats in the NBM + MSA group showed normal posture, eating, and drinking by the third day after surgery.

Because the behavioral data for the CON groups from both the IBO and RF sections of the experiment were not significantly different, they were combined, and all of the behavioral data were analyzed together in a three-way analysis of variance (ANOVA). The percentage of total responses that were correct for each test session was averaged into 2-day blocks for the analysis.

Stem discrimination. CON rats continued criterion level choice accuracy (Fig. 4). All six groups of rats with lesions also continued criterion level choice accuracy. No main effect or interaction was significant $(F<1)$.

Arm discrimination. CON and DGP rats had only a small transient decrease in choice accuracy (Fig. 5). They returned to criterion performance in a mean of 3.8 test sessions. At the start of postoperative testing, NBM, MSA, and NBM + MSA groups had significant decreases in choice accuracy. These rats relearned the task and reached criterion. The mean number of trials to criterion for each group of rats was: NBM-IBO, 9.6; NBM-RF, 9.8; MSA-IBO, 13.4; MSA-RF, 15.8; NBM + MSA, 13.8 (see Fig. 4).

ANOVA showed that choice accuracy for all groups improved during testing $(F(8,764)=354.33, p<0.01)$. The choice accuracy for rats with lesions in the NBM, MSA, and NBM + MSA was impaired relative to that of $\operatorname{CON}$ rats $(F(6,764)=798.54, p<0.01)$. The choice accuracy of rats with MSA lesions was impaired relative to that of rats with NBM lesions $(\rho<0.05)$. A group by test session effect $(F(48,764)=71.01, p<0.01)$ and Sheffe contrasts revealed that the choice accuracy of rats with NBM, MSA, and NBM + MSA lesions was significantly impaired relative to that of CON rats for blocks 1 through 7 inclusive $(p<0.05)$. The choice accuracy of rats with MSA and NBM + MSA lesions was impaired relative to that of rats with NBM lesions for blocks 3 through 7 , inclusive $(\rho<0.05)$. No significant difference in choice accuracy was found between the MSA and NBM + MSA groups.

Rats with RF lesions in the NBM and MSA had impaired choice accuracy relative to rats with IBO lesions in these areas $(F(1,622)$ $=5.13, p<0.05)$. A lesion by test session effect $(F(8,622)=$ 14.16, $p<0.01$ ) and Sheffe contrasts revealed that the rats in the NBM-RF and MSA-RF groups had impaired choice accuracy relative to rats in the NBM-IBO and MSA-IBO groups for blocks 3 through 7 inclusive $(p<0.05)$.

Finally, a lesion by group by test session effect was found $(F(16$, $622)=4.19, p<0.01$ ). This interaction was due to the MSA-RF lesion group, which had impaired choice accuracy relative to the MSA-IBO lesion group for blocks 4 through 7 inclusive $(p<0.05)$.

Reversal of stem discrimination. For the first few trials of the reversed stem discrimination, every rat made errors in the stem. All rats quickly learned the reversal and reached criterion. The CON group reached the criterion level in a mean of 4.2 test sessions. The mean number of trials to criterion for each lesion group was: DGP, 4.1; NBM-IBO, 4.6; NBM-RF, 4.4; MSA-IBO, 4.9; MSA-RF, 5.0; NBM + MSA, 10.5). Choice accuracy of the rats in the NBM + MSA group differed significantly from that of CON group $(F(6,511)=36.00, p$ 


\section{DGP IBOTENIC ACID}

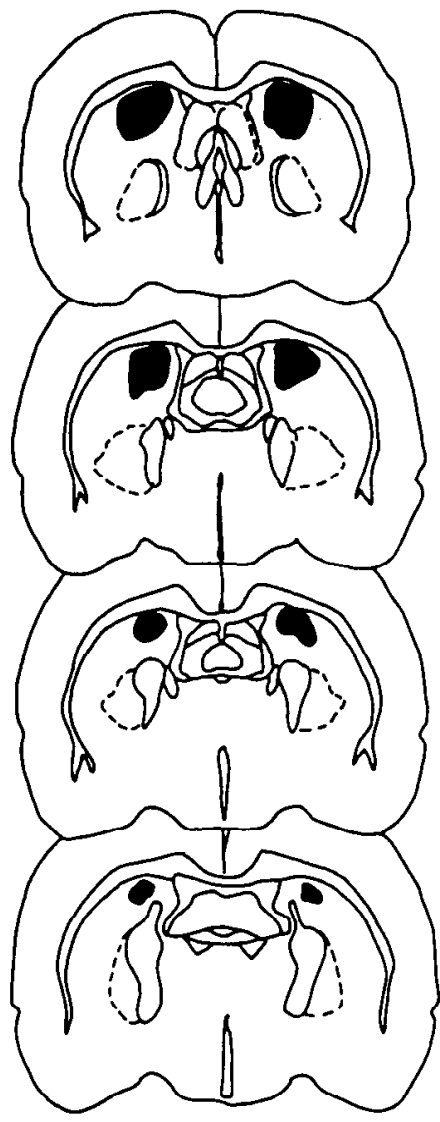

Figure 3. Drawings of coronal sections showing a typical IBO lesions of the DGP. Shaded areas indicate areas of cell loss.

$<0.01)$. With the exception of test sessions $(F(5,511)=44.49, p$ $<0.01$ ), no other main effect or interaction was significant $(F<1)$.

\section{Extended delay}

Stem discrimination. With the initial delay of $5 \mathrm{sec}$, choice accuracy of rats in both the CON and NBM + MSA groups was at or above the criterion level (see Fig. 5). However, when the delay was increased to $10 \mathrm{~min}$, the choice accuracy of rats in the NBM + MSA group was sightly, but significantly, impaired relative to the performance of rats in the CON group $(F(1,52)=4.29, p<0.05)$ and did not improve during the 18 test sessions with the 10-min delay. When the delay was decreased to $5 \mathrm{sec}$, the mean number of trials for choice accuracy to return to criterion for rats with CON lesions (1.6) was not significantly different from the number required for rats with lesions (1.9) (Fig. 6).

Arm discrimination. With the initial delay of $5 \mathrm{sec}$, the choice accuracy of rats in the CON and NBM + MSA groups was also at or above the criterion level. However, with the 10-min delay, the choice accuracy of rats in both groups was substantially decreased relative to their performance with the 5 -sec delay $(F(1,52)=21.2$, $p<0.01)$. Of greater importance, the choice accuracy of rats in the $\mathrm{NBM}+\mathrm{MSA}$ group was markedly impaired relative to the performance of rats in the CON group $(F(1,52)=33.28, p 0.01)$. The choice accuracy of rats in both these groups did not improve during the 18 test sessions. When the delay was decreased to $5 \mathrm{sec}$, the mean number of trials for choice accuracy to return to criterion for rats with CON lesions (1.7) was not significantly different from the number required for rats with lesions (2.1).

\section{Discussion}

The behavioral effects of lesions in either the NBM or the MSA were similar in all three behavioral tasks. Choice accuracy was (1) impaired in the retention of the trial-dependent discrimination on the arms of the T-maze, (2) unimpaired in the retention of the trialindependent discrimination on the stem of the T-maze, and (3) unimpaired in the reversal of this discrimination (except for the NBM + MSA group which was slightly impaired). The impairment in the ability to choose the correct arm indicates that both of these brain structures normally mediate choice accuracy in this type of task. The frontal cortex and hippocampus may form a closely interrelated system for processing of different types of information about memory (Mishkin, 1982).

The pattern of behavioral changes observed here suggests that the rats suffered a specific impairment in memory. In order to perform the stem discrimination accurately, the rat had to identify the location in the maze where it had been placed, determine right from left, and respond correctly. Therefore, perceptual, motivational, and motoric processes were intact. What was not intact was the rat's ability to remember short-term, trial-specific information. This trial-dependent memory deficit cannot be attributed to a general retrograde amnesia because the lesioned rats were unimpaired in the reacquisition of the stem discrimination. Furthermore, the dissociation of performance between the arm and stem discriminations cannot be attributed to a difference in the relative difficulty of the two tasks because the rats learned both discriminations at approximately the same rate. Thus, the pattern of dissociations suggests that rats with NBM, MSA, or NBM + MSA lesions had an amnesic syndrome with a specific impairment in trial-dependent (working) memory.

During the initial preoperative testing with the 5-sec delay between the forced run and the choice run in the arm discrimination, all rats with lesions in either the NBM or MSA had impaired choice accuracy. However, all rats eventually reached criterion levels of accuracy. This improvement in performance did not reflect a complete recovery of function, however. When the memory requirement of the task was emphasized by increasing the delay from $5 \mathrm{sec}$ to $10 \mathrm{~min}$, the impairment in the lesioned rats returned. This sensitivity of choice accuracy to the delay interval is additional support for the interpretation that these lesions produced a specific impairment in memory (Mishkin, 1982). All of the components of the task were the same at the long delay as at the short delay except for the length of time the forced run had to be remembered. Consequently, all those processes necessary for normal performance at the short delay must have been intact, and the most likely explanation for the impairment at the longer delay involves the psychological processes that were related to the delay interval-processes generally interpreted as memory (Mishkin, 1982). This interpretation is compromised slightly by the impairment on the stem discrimination during the extended delay. However, the magnitude of the deficit on the arm discrimination was much greater than that in the stem discrimination. The slight impairment in the stem discrimination may have been due to interference from the reversal of the stem discrimination that occurred just prior to the testing with the extended delay.

The recovery of behavioral performance with the short delay interval between the forced run and choice run may have been due to compensatory biochemical changes made by the brain in an attempt to adjust to the localized damage. Immediately after lesions in the basal forebrain, sodium-dependent high affinity choline uptake (Wenk and Olton, 1984) and 2-deoxyglucose uptake (London et al., 1984) were both decreased in the frontal cortex and hippocampus. Six weeks after the lesions both measures returned to control levels. Postsynaptic muscarinic receptors in the neocortex become supersensitive following basal forebrain lesions (McKinney and Coyle, 1982). Low affinity sites decreased immediately after the lesion and returned to normal in 3 weeks, whereas high affinity sites were increased chronically after the lesion. Therefore, the behavioral recovery observed with the short delay may have been due to 

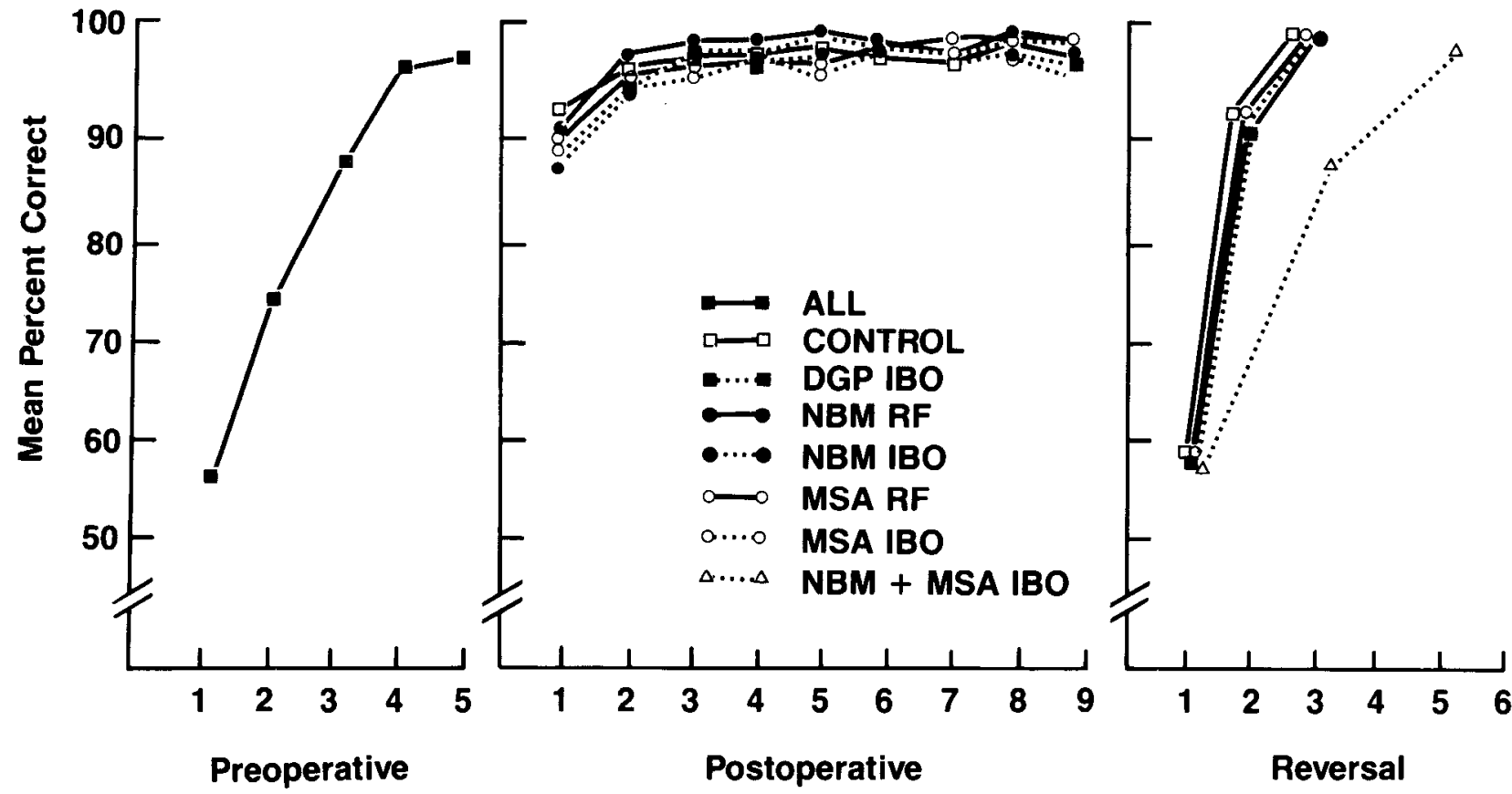

\section{Blocks of Two Test Sessions}

Figure 4. Summary of the behavioral data from the stem discrimination. The vertical axis presents the mean percentage of correct choices. The horizontal axis presents blocks of Iwo lest sessions. Preoperatively, all rats learned the stem discrimination within 10 test sessions. Postoperatively, the choice accuracy on the stem was unimpaired for all groups of rats. All groups of rats acquired the stem reversal at the same rate, reaching criterion by the fifth test session (except for the NBM + MSA group).

\section{Arm Discrimination}
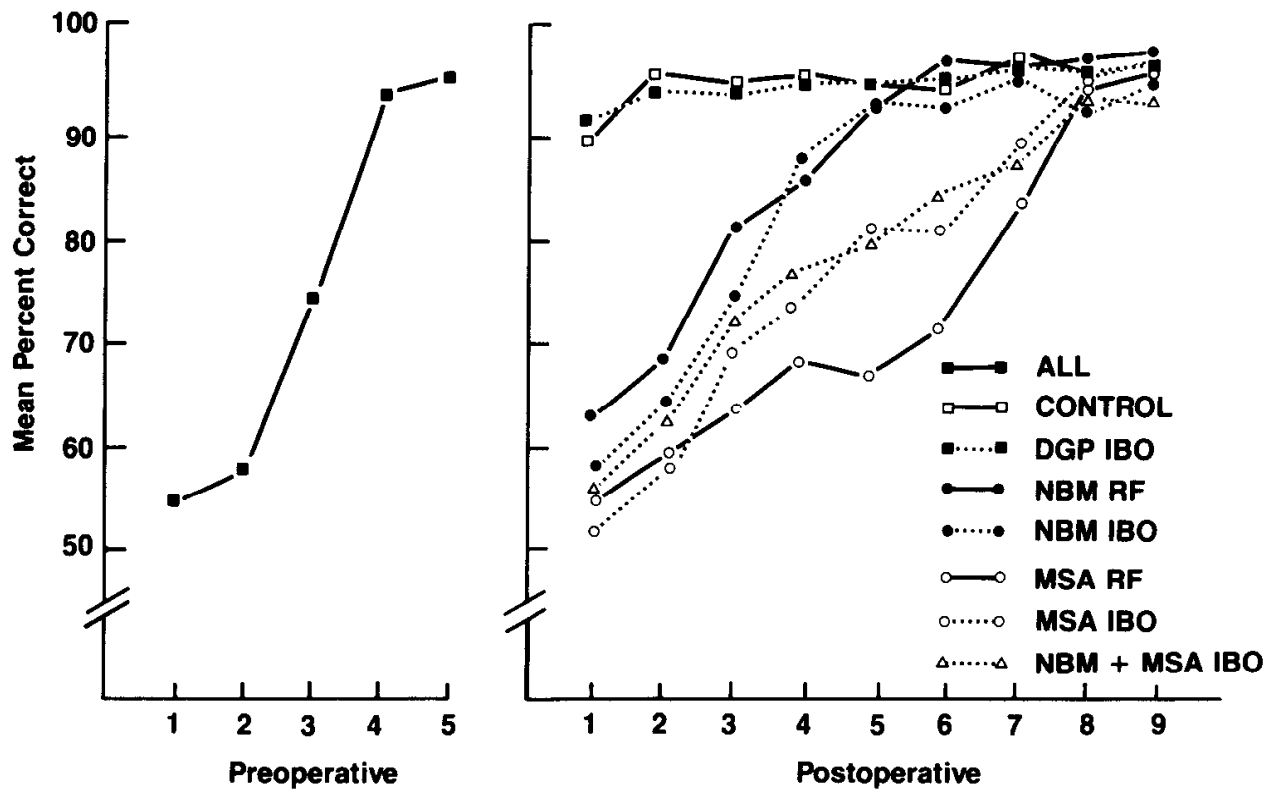

Figure 5. Summary of behavioral data from the arm discrimination. The vertical axis presents the mean percentage of correct choices. The horizontal axis presents blocks of two test sessions. Preoperatively, all rats learned the arm discrimination within 10 test sessions. Postoperatively, the choice accuracy on the arms was impaired during the first several blocks of test sessions following NBM and MSA lesions. Choice accuracy rapidly improved to criterion levels.

Blocks of Two Test Sessions

increased activity of the cholinergic neurons that survived the lesion, restored glucose utilization in the neocortex, or compensatory changes in postsynaptic muscarinic receptors. However, these biochemical changes were not sufficient to re-establish normal behavior because an impairment persisted with the long delay.

The impairment of choice accuracy in the arms was probably due to changes in the cholinergic system. The majority of the cells within the NBM are cholinergic (Mesulam et al., 1983; Armstrong et al., 1984; Saper, 1984). Furthermore, injections of IBO in the NBM with techniques similar to those used here reduced cholinergic markers in the neocortex but did not affect other neurochemical systems, including GABAergic neurons intrinsic to cortex as well as noradre- 


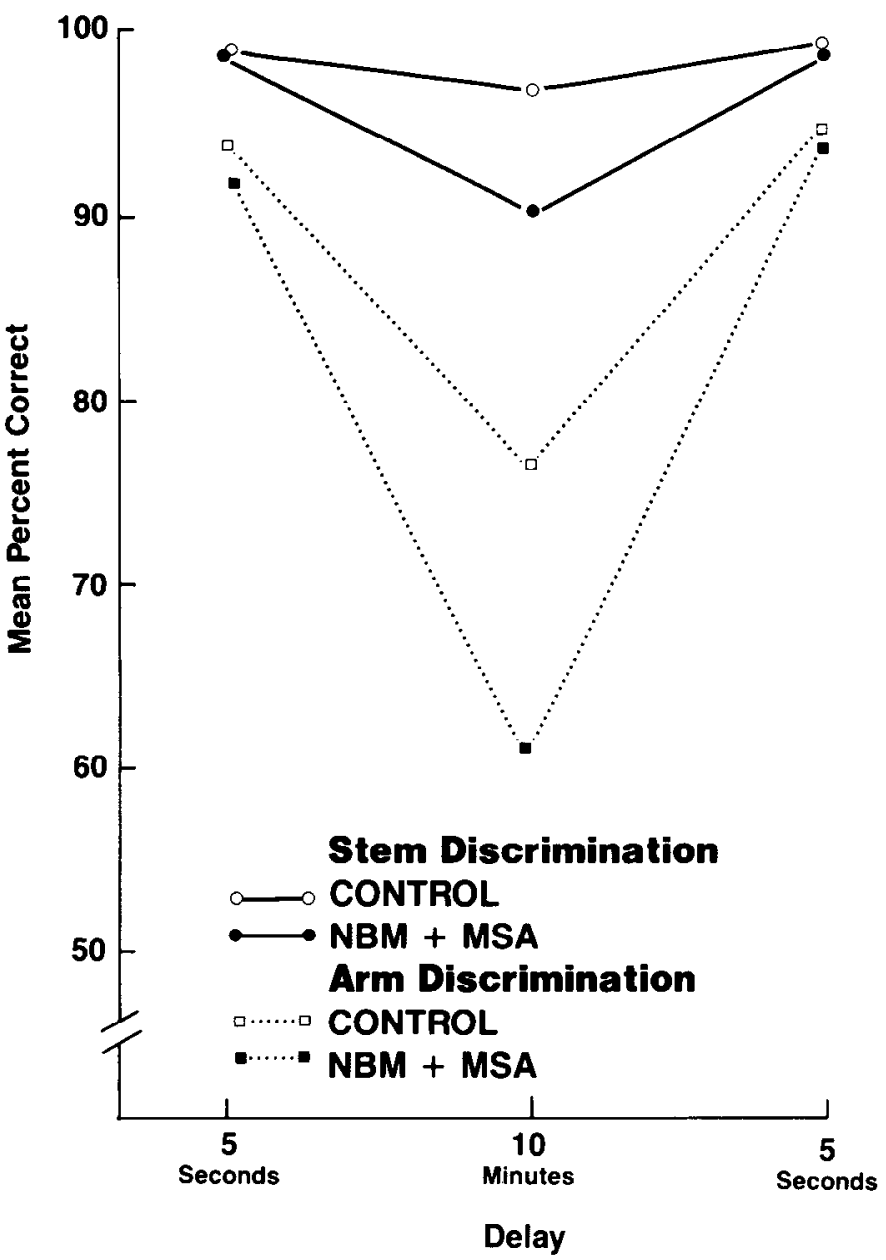

Figure 6. Summary of behavioral data from the extended delay task. The vertical axis presents the mean percentage of correct choices. The horizontal axis presents the different delays (6 test sessions for each of the 5-sec delays, 18 test sessions for the 10-min delay). At the 5-sec delay, all rats performed at or above criterion level of performance on both the stem and arm discriminations. At the 10-min delay, the choice accuracy of the NBM + MSA group was slightly impaired relative to that of the CON group in the stem discrimination, and greatly impaired in the arm discrimination. All rats quickly returned to the criterion level of performance when the delay was decreased to $5 \mathrm{sec}$.

nergic, serotonergic, and histaminergic afferents (Johnston et al., 1979,1981 ). Similar neurochemical data are not available following injections of IBO into the MSA. However, anticholinergic drugs produced an impairment of choice accuracy in memory tasks that required trial-dependent memory (Okaichi and Jarrard, 1982; Wirsching et al., 1984). Individual assessment of all other transmitter systems is necessary before any final conclusions can be drawn. In any case, the similar behavioral effects produced by the RF and IBO lesions show that the cell bodies themselves (whatever their transmitter) in the NBM and MSA are functionally important for normal choice accuracy in the arm discrimination; damage to the fibers of passage in these areas is not necessary to obtain memory impairments

Lesions in either the NBM or MSA produced similar behavioral deficits. However, this result was not due to similar effects on ChAT levels. Lesions in the NBM significantly decreased ChAT levels in the frontal cortex but not the dorsal or ventral hippocampus, whereas lesions in the MSA produced the opposite effect (McKinney et al., 1983; Wenk et al., 1984). Consequently, the lesions themselves were clearly distinct in terms of both their placement and their effect on cholinergic projections.

The large difference in ChAT levels between the control groups of the various sections of the present study was probably due to the different times that the rats were obtained from the breeders. ChAT levels and sodium-dependent high affinity choline uptake have varied between groups of rats obtained at different times from the same breeder (unpublished observations). Such variability does not affect the interpretation of the ChAT data within each section, because appropriate control rats were included from each shipment.

DGP lesions had no significant effect on choice accuracy in any of the discriminations, nor did they significantly reduce ChAT levels in the frontal cortex or hippocampus. These results are important because they demonstrate that the behavioral changes following injections of IBO into the NBM and MSA were due to selective damage of neuronal cell bodies located in those areas, rather than to IBO damage to the brain or destruction of the contiguous portion of the globus pallidus.

Clinical and experimental observations with people indicate that the cholinergc system is importantly involved in memory (Corkin, 1981). Patients with Alzheimer's disease consistently show degeneration of central cholinergic systems (Davies and Maloney, 1976; Davies, 1979; Perry et al., 1977). The NBM and MSA lesions in this experiment produce some of the final biochemical changes observed in these patients. The pattern of behavioral results are consistent with previous suggestions that such pathological changes in the basal forebrain cholinergic system are sufficient to produce memory impairments (Davis and Yamamura, 1978; Perry et al., 1978; Coyle et al., 1983).

\section{References}

Armstrong, D. M., C. B. Saper, A. I. Levey, B. H. Wainer, and R. D. Terry (1983) Distribution of cholinergic neurons in the rat brain: Demonstrated by the immunocytochemical localization of choline acetyltransferase. J. Comp. Neurol. 216: 53-68.

Bartus, R. T. (1980) Cholinergic drug effects on memory and cognition in animals. In Aging in the 80's: Psychological /ssues. L. W. Poon, ed., pp. 163-180, American Psychological Association, Washington, D. C

Bartus, R. T., R. L. Dean, B. Beer, and A. S. Lippa (1982) The cholinergic hypothesis of geriatric memory dysfunction: A critical review. Science 47 408-417.

Bigl, V., N. J. Woolf, and L. L. Butcher (1982) Cholinergic projections from the basal forebrain to frontal, parietal, temporal, occipital, and cingulate cortices: A combined fluorescent tracer and acetylcholinesterase. Brain Res. Bull. 8: 724-749.

Corkin, S. (1981) Acetylcholine, aging, and Alzheimer's disease: Implications for treatment. Trends Neurosci. 4: 287-290.

Coyle, J. T., and R. Schwarcz (1983) The use of excitatory amino acids as selective neurotoxins. In Handbook of Chemical Neuroanatomy. Vol. 1: Methods in Chemical Neuroanatomy, A. Bjorklund and T. Hokfelt, eds., pp. 508-527, Elsevier Science Publishers, New York.

Coyle, J. T., D. L. Price, and M. R. DeLong (1983) Alzheimer's disease: A disorder of cortical cholinergic innervation. Science 219: 1184-1190.

Davies, P. (1979) Neurotransmitter-related enzymes in senile dementia of the Alzheimer type. Brain Res. 171: 319-327.

Davies, P., and A. J. F. Maloney (1976) Selective loss of cholinergic neurons in Alzheimer's disease. Lancet 2: 1403

Davis, K. L., and H. I. Yamamura (1978) Cholinergic underactivity in human memory disorders. Life Sci 23: 1729-1734.

Flicker, C., R. L. Dean, D. L. Watkins, S. K. Fisher, and R. T. Bartus (1983) Behavioral and neurochemical effects following neurotoxic lesions of a major cholinergic input to the cerebral cortex in the rat. Pharmacol. Biochem. Behav. 18: 973-981.

Fonnum, F. (1975) A rapid radiochemical method for the determination of choline acetyltransferase. J. Neurochern. 24: 407-409.

Hartgraves, S. L., P. L. Mensah, and P. H. Kelly (1982) Regional decreases of cortical choline acetyltransferase after lesions of the septal area and in the area of nucleus basalis magnocellularis. J. Neurosci. Methods 7:23692376.

Johnston, M. V., M. McKinney, and J. T. Coyle (1979) Evidence for a cholinergic projection to neocortex from neurons in the basal forebrain. Proc. Natl. Acad. Sci. U. S. A. 76: 5392-5396.

Johnston, M. V., M. McKinney, and J. T. Coyle (1981) Neocortical cholinergic innervation in the rat. Exp. Brain Res. 43: 159-172. 
Krieg, W. J. S. (1946) Connections of the cerebral cortex. I. The albino rat A. Topography of the cortical areas. J. Comp. Neurol. 84: 221-275.

Lehmann, J., J. I. Nagy. S. Atmadja, and H. C. Fibiger (1980) The nucleus basalis magnocellularis: The origin of a cholinergic projection to the neocortex of the rat. J. Neurosci. Methods 5: 1161-1174.

LoContc, G., L. Bartolini, F. Casamenti, I. Marconcini-Pepeu, and G. Pepeu (1982) Lesions of cholinergic forebrain nuclei: Changes in avoidance behavior and scopolamine actions. Pharmacol. Biochem. Behav. 17: 933937.

London, E., M. McKinney, M. Dam, A. Ellis, and J. T. Coyle (1984) Decreased cortical glucose utilization after ibotenate lesion of the rat ventromedial globus pallidus. Brain Res., in press.

Lowry, O. H., N. J. Rosebrough, A. L. Farr, and R. J. Randall (1951) Protein measurement with Folin phenol reagent. J. Biol. Chem. 193: 265-275.

McKinney, M., and J. T. Coyle (1982) Regulation of neocortical muscarinic receptors: Effects of drug treatment and lesions. J. Neurosci. 2: 97-105.

McKinney, M., J. T. Coyle, and J. C. Hedreen (1983) Topographic analysis of the innervation of the rat neocortex and hippocampus by the basal forebrain cholinergic system. J. Comp. Neurol. 217: 103-121.

Mesulam, M. -M., E. J. Mufson, A. I. Levey, and B. H. Wainer (1983) Cholinergic innervation of cortex by the basal forebrain: Cytochemistry and cortical connections of the septal area, diagonal band nuclei, nucleus basalis (substantia innominate) and hypothalamus in the rhesus monkey. J. Comp. Neurol. 214: 170-197.

Mishkin, M. (1982) A memory system in the monkey. Philos. Trans. R. Soc. Lond. (Biol) 298: 85-95.

Mitchell, S. J., J. N. P. Rawlins, O. Steward, and D. S. Olton (1982) Medial septal area lesions disrupt theta rhythm and cholinergic staining in medial entorhinal cortex and-produce impaired radial arm maze behavior in rats J. Neurosci. 2: 292-302.

Okaichi, H., and L. E. Jarrard (1982) Scopolamine impairs performance of a place and cue task in rats. Behav. Neural Biol. 35: 319-325.

Pearson, R. C. A., K. C. Gatter, and T. P. S. Powell (1983) The cortical relationships of certain basal ganglia and cholinergic basal forebrain nuclei. Brain Res. 261: 327-330.

Perry, E. K., R. H. Perry, G. Blessed, and B. E. Tomlinson (1977) Necropsy evidence of central cholinergic deficits in senile dementia. Lancet 1: 189.

Perry, E. K., B. E. Tomlinson, G. Blessed, K. Bergmann, P. H. Gibson, and R. H. Perry (1978) Correlation of cholinergic abnormalities with senile plaques and mental test scores in senile dementia. Br. Med. J. 2: 14571459.

Saper, C. B. (1984) Organization of cerebral cortical afferent systems in the rat. I. Magnocellular basal nucleus. J. Comp. Neurol. 222: 313-342.

Sofroniew, M. V., F. Eckenstein, H. Thoenen, and A. C. Cuello (1982) Topography of choline acetyltransferase-containing neurons in the forebrain of the rat. Neurosci. Lett. 33: 7-12.

Wenk, G. L., and D. S. Olton (1984) Recovery of neocortical choline acetyltranslerase activity following ibotenic acid injection into the nucleus basalis of Meynert in rats. Brain Res. 293: 184-186.

Wenk, G. L., B. Cribbs, and L. McCall (1984) Nucleus basalis magnocellularis: Optimal coordinates for selective reduction of choline acetyltransferase in frontal neocortex by ibotenic acid injections. Exp. Brain Res. 56: 335340.

Wirsching, B. A., R. J. Beninger, K. Jhamandas, R. J. Boegman, and S. R. El-Defrawy (1984) Differential effects of scopolamine on working and reference memory of rats in the radial maze. Pharmacol. Biochem. Behav. 20: 659-662. 